\title{
Exposing the quantum geometry of spin-orbit-coupled Fermi superfluids
}

\author{
M. Iskin \\ Department of Physics, Koç University, Rumelifeneri Yolu, 34450 Sartyer, Istanbul, Turkey
}

(Received 22 November 2017; published 28 June 2018)

\begin{abstract}
The coupling between a quantum particle's intrinsic angular momentum and its center-of-mass motion gives rise to the so-called helicity states that are characterized by the projection of the spin onto the direction of momentum. In this paper, by unfolding the superfluid-density tensor into its intrahelicity and interhelicity components, we reveal that the latter contribution is directly linked with the total quantum metric of the helicity bands. We consider both Rashba and Weyl spin-orbit couplings across the BCS-BEC crossover and show that the geometrical interhelicity contribution is responsible for up to a quarter of the total superfluid density. We believe this is one of those elusive effects that may be measured within the highly tunable realm of cold Fermi gases.
\end{abstract}

DOI: 10.1103/PhysRevA.97.063625

\section{INTRODUCTION}

The earliest association between the physical property of a quantum system and the topological structure of its underlying Bloch bands was made [1] soon after the realization that the observed quantization of Hall conductivity was nothing but conclusive evidence for its quantum geometric origins. In particular, it turns out that the integral of the Berry curvature (defined just below) in two-dimensional electron systems is a topological invariant of the system and that its quantized value is proportional to the Hall conductivity of a band insulator. Bearing this hindsight in mind, not only the topological but also the geometrical structure of the Bloch bands has been playing an ever-increasing role in modern quantum physics [2-9], where more and more physical phenomena are proposed to find their roots in the so-called geometric quantum mechanics [10].

In order to specify these topological and geometrical ideas one needs to introduce the quantum geometric tensor of a given Bloch band [1,11], which is also referred to as the Fubini-Study metric tensor in the broader context of differential geometry. For this purpose, let us consider a Bloch Hamiltonian density $H_{0 \mathbf{k}}$ and label its single-particle energy eigenvalues $\epsilon_{i \mathbf{k}}$ and energy eigenstates $|i \mathbf{k}\rangle$ by the band index $i$ and momentum $\mathbf{k}=\sum_{\nu} k_{v} \hat{\boldsymbol{v}}$ in the $D$-dimensional Brillouin zone with coordinates $v=\{x, y, z\}$. Since the gaugeinvariant quantum geometric tensor of the $i$ th band $Q_{i \mathbf{k}}^{\mu \nu}=$ $\left\langle\partial_{k_{\mu}} i \mathbf{k} \mid \partial_{k_{\nu}} i \mathbf{k}\right\rangle-\left\langle\partial_{k_{\mu}} i \mathbf{k} \mid i \mathbf{k}\right\rangle\left\langle i \mathbf{k} \mid \partial_{k_{\nu}} i \mathbf{k}\right\rangle$ is a complex one, it is customary to divide it into two gauge-invariant tensors as $Q_{i \mathbf{k}}^{\mu \nu}=g_{i \mathbf{k}}^{\mu \nu}-(i / 2) F_{i \mathbf{k}}^{\mu \nu}[1,11]$, where $g_{i \mathbf{k}}^{\mu \nu}$ is the so-called quantum metric and $F_{i \mathbf{k}}^{\mu \nu}$ the Berry curvature. Even though the Berry curvature is omnipresent in nature and most of the measurable quantum geometric effects have so far been related to it [1-9], the importance of the quantum metric is yet to be recognized in the light of recent theoretical proposals on a diverse range of problems in condensed-matter physics [12-24]. Among them, the most recent connection between the quantum metric of the noninteracting Bloch bands and the superfluid (SF) weight tensor, i.e., in the context of multiband attractive Hubbard models, stands out as an important milestone for our fundamental understanding of superfluidity and superconductivity [21-25]. For instance, in marked contrast with the single-flat-band systems where superfluidity is strictly forbidden, it may succeed in a flat band in the presence of other bands (e.g., the Lieb lattice) as a direct result of the geometric effects through interband tunnelings [21,22]. In addition, the geometrical contribution to the supercurrent [23] also gives further insight into the baffling controversy around the superconductivity of graphene without supercurrent in the vicinity of its Dirac points [26,27].

Motivated by these theoretical proposals [21-25], here we explore the experimental feasibility of its counterpart effect in the SF density of spin-orbit-coupled Fermi gases [28-31], for which the coupling between the intrinsic spin and orbital motion gives rise to the so-called helicity states that are characterized by the projection of the spin onto the direction of momentum. For this purpose, we first split the SF-density tensor into two contributions depending on their physical origin, i.e., while the intrahelicity contribution has the conventional form [32] determined solely by the corresponding helicity spectrum and takes the real intraband processes into account, the interhelicity one accounts for the virtual interband processes and is directly linked with the total quantum metric of the helicity bands. We then consider both Rashba and Weyl spin-orbit couplings (SOCs) across the BCS-BEC crossover and show that the geometrical interhelicity contribution is responsible for up to a quarter of the total SF density. Given the recent realizations of two-dimensional (2D) SOCs in atomic Bose and Fermi gases [33-36], measuring the quantum geometry of their helicity bands would endorse the elusive quantum metric to the level of, and with arguably as far-reaching impact on modern physics as, the Berry curvature.

\section{BCS MEAN-FIELD THEORY}

Assuming a pseudospin- $1 / 2$ Fermi SF with an equal number of $\uparrow$ and $\downarrow$ components, we start with the BCS mean-field description of stationary Cooper pairs with zero center-of-mass momentum. A compact way to write this model Hamiltonian 
(in units of $\hbar=1$ ) is [28-31,37]

$$
\begin{aligned}
H= & \frac{1}{2} \sum_{\mathbf{k}} \Psi_{\mathbf{k}}^{\dagger}\left(\begin{array}{cc}
\xi_{\mathbf{k}} \sigma_{0}+\mathbf{d}_{\mathbf{k}} \cdot \boldsymbol{\sigma} & i \Delta \sigma_{y} \\
-i \Delta \sigma_{y} & -\xi_{\mathbf{k}} \sigma_{0}+\mathbf{d}_{\mathbf{k}} \cdot \boldsymbol{\sigma}^{*}
\end{array}\right) \Psi_{\mathbf{k}} \\
& +\sum_{\mathbf{k}} \xi_{\mathbf{k}}+\frac{\Delta^{2}}{U},
\end{aligned}
$$

where the spinor operators $\Psi_{\mathbf{k}}^{\dagger}=\left(\psi_{\mathbf{k}}^{\dagger} \psi_{-\mathbf{k}}\right)$ with $\psi_{\mathbf{k}}^{\dagger}=$ $\left(\psi_{\uparrow \mathbf{k}}^{\dagger} \psi_{\downarrow \mathbf{k}}^{\dagger}\right)$ create $\sigma=\{\uparrow, \downarrow\}$ fermions with $\pm \mathbf{k}$ momentum, the shifted dispersion $\xi_{\mathbf{k}}=\epsilon_{\mathbf{k}}-\mu$ describes a free Fermi gas with the single-particle energy $\epsilon_{\mathbf{k}}=k^{2} / 2 m$ and the chemical potential $\mu$, and the BCS mean field $\Delta=U\left\langle\psi_{\uparrow \mathbf{k}} \psi_{\downarrow-\mathbf{k}}\right\rangle$ is taken as a real parameter without the loss of generality. Here $U \geqslant 0$ is the strength of the contact interaction and $\langle\cdots\rangle$ denotes the thermal average. Furthermore, $\sigma_{0}$ is the $2 \times 2$ identity matrix and $\sigma=\sum_{\nu} \sigma_{v} \hat{\boldsymbol{v}}$ is a vector of Pauli spin matrices in such a way that $\mathbf{d}_{\mathbf{k}}=\sum_{\nu} \alpha_{\nu} k_{v} \hat{\boldsymbol{v}}$ corresponds to a Weyl SOC when $\alpha_{v}=\alpha$ for all $v=\{x, y, z\}$ and to a Rashba SOC when $\alpha_{z}=0$. Here $\hat{\boldsymbol{v}}$ is a unit vector along the $v$ direction and we choose $\alpha \geqslant 0$ without loss of generality.

Since this Hamiltonian and its numerous variations have been well studied in the recent cold-atom literature, we simply quote the self-consistency equations for $\Delta$ and $\mu$ [28-31,37],

$$
\begin{gathered}
\frac{1}{U}=\frac{1}{2 \mathcal{V}_{D}} \sum_{s \mathbf{k}} \frac{\mathcal{X}_{s \mathbf{k}}}{2 E_{s \mathbf{k}}}, \\
n=\frac{1}{2 \mathcal{V}_{D}} \sum_{s \mathbf{k}}\left(1-\frac{\xi_{s \mathbf{k}}}{E_{s \mathbf{k}}} \mathcal{X}_{s \mathbf{k}}\right),
\end{gathered}
$$

where $\mathcal{V}_{D}$ corresponds to the area $A$ in two dimensions and volume $V$ in three dimensions, $s= \pm$ labels the helicity bands, $\mathcal{X}_{s \mathbf{k}}=\tanh \left(E_{s \mathbf{k}} / 2 T\right)$ is a thermal factor with the Boltzmann constant $k_{B}$ set to unity and $T$ the temperature, $\xi_{s \mathbf{k}}=\epsilon_{s \mathbf{k}}-\mu$ is the shifted dispersion for the $s$-helicity band with $\epsilon_{s \mathbf{k}}=$ $\epsilon_{\mathbf{k}}+s d_{\mathbf{k}}$ and the strength of the SOC $d_{\mathbf{k}}=\left|\mathbf{d}_{\mathbf{k}}\right|$, and $E_{s \mathbf{k}}=$ $\left(\xi_{s \mathbf{k}}^{2}+\Delta^{2}\right)^{1 / 2}$ is the energy spectrum of the quasiparticles for the corresponding helicity band. Here the number Eq. (3) for the density $n=N / \mathcal{V}_{D}$ of particles follows from $N=$ $\sum_{\mathbf{k}}\left\langle\psi_{\mathbf{k}}^{\dagger} \psi_{\mathbf{k}}\right\rangle$. Given that the model Hamiltonian is effectively a two-band one with a single $\mathbf{k}$-independent order parameter and the time-reversal symmetry is also manifest, we argue in this paper that spin-orbit-coupled Fermi SFs may promise one of the ideal test beds for the exploration of the recently proposed quantum geometric effects [21-25].

\section{SUPERFLUID-DENSITY TENSOR}

As a counterpart to the geometric effects in the SF-weight tensor of multiband attractive Hubbard models [21-24], here we study the SF-density tensor $\rho_{\mu \nu}$ of a continuum model in the context of spin-orbit-coupled Fermi gases. Following Refs. [23-25], we unravel the intrahelicity and interhelicity contributions to $\rho_{\mu \nu}=\rho_{\mu \nu}^{\text {intra }}+\rho_{\mu \nu}^{\text {inter }}$ as

$$
\begin{aligned}
& \rho_{\mu \nu}^{\text {intra }}=\frac{m \Delta^{2}}{2 \mathcal{V}_{D}} \sum_{s \mathbf{k}}\left(\frac{\mathcal{X}_{s \mathbf{k}}}{E_{s \mathbf{k}}^{3}}-\frac{\mathcal{Y}_{s \mathbf{k}}}{2 T E_{s \mathbf{k}}^{2}}\right) \frac{\partial \xi_{s \mathbf{k}}}{\partial k_{\mu}} \frac{\partial \xi_{s \mathbf{k}}}{\partial k_{v}}, \\
& \rho_{\mu \nu}^{\text {inter }}=-\frac{m \Delta^{2}}{\mathcal{V}_{D}} \sum_{s \mathbf{k}} \frac{d_{\mathbf{k}} \mathcal{X}_{s \mathbf{k}}}{s \xi_{\mathbf{k}} E_{s \mathbf{k}}} g_{\mathbf{k}}^{\mu \nu}
\end{aligned}
$$

where $\mathcal{Y}_{s \mathbf{k}}=\operatorname{sech}^{2}\left(E_{s \mathbf{k}} / 2 T\right)$ is a thermal factor and $g_{\mathbf{k}}^{\mu \nu}=$ $\sum_{s} g_{s \mathbf{k}}^{\mu \nu}$ is the total quantum metric of the helicity bands. Here, while $\rho_{\mu \nu}^{\text {intra }}$ is finite unless $\Delta=0, \rho_{\mu \nu}^{\text {inter }}$ is finite unless $\alpha=0$ together with $\Delta=0$.

We recall that the quantum metric of a given Bloch band is generally defined by the energy spectrum $\epsilon_{i \mathbf{k}}$ of the Hamiltonian and its corresponding eigenfunctions $|i \mathbf{k}\rangle$ in a highly nontrivial way $[1,11]$. This can be illustrated by combining the generic definition of the metric $g_{i \mathbf{k}}^{\mu \nu}=\operatorname{Re}\left[\left\langle\partial_{k_{\mu}} i \mathbf{k}\right|(\mathbb{I}-\right.$ $\left.|i \mathbf{k}\rangle\langle i \mathbf{k}|)\left|\partial_{k_{v}} i \mathbf{k}\right\rangle\right]$ for the $i$ th band together with the completeness relation $\mathbb{I}=\sum_{i}|i \mathbf{k}\rangle\langle i \mathbf{k}|$ for a given $\mathbf{k}$ state, leading to an equivalent but numerically much more practical expression $g_{i \mathbf{k}}^{\mu \nu}=\operatorname{Re} \sum_{j\{\neq i\}}\left\langle i \mathbf{k}\left|\partial_{k_{\mu}} H_{0 \mathbf{k}}\right| j \mathbf{k}\right\rangle\left\langle j \mathbf{k}\left|\partial_{k_{v}} H_{0 \mathbf{k}}\right| i \mathbf{k}\right\rangle /\left(\epsilon_{i \mathbf{k}}-\right.$ $\left.\epsilon_{j \mathbf{k}}\right)^{2}$. In the particular application to our model, the single-particle problem is determined by the wave equation $H_{0 \mathbf{k}}|s \mathbf{k}\rangle=\epsilon_{s \mathbf{k}}|s \mathbf{k}\rangle$, where $H_{0 \mathbf{k}}=\epsilon_{\mathbf{k}} \sigma_{0}+\mathbf{d}_{\mathbf{k}} \cdot \sigma$ is the Hamiltonian density, giving rise to two helicity bands indexed by $s= \pm$ as long as $\alpha \neq 0$. Thus, we find $g_{+, \mathbf{k}}^{\mu \nu}=g_{-, \mathbf{k}}^{\mu \nu}$, so $g_{\mathbf{k}}^{\mu \nu}=$ $\alpha_{\mu} \alpha_{v}\left(d_{\mathbf{k}}^{2} \delta_{\mu \nu}-\alpha_{\mu} \alpha_{\nu} k_{\mu} k_{v}\right) / 2 d_{\mathbf{k}}^{4}$ with $\delta_{i j}$ the Kronecker delta, and also the total quantum metric can also be represented as $g_{\mathbf{k}}^{\mu \nu}=\partial_{k_{\mu}} \hat{\mathbf{d}}_{\mathbf{k}} \cdot \partial_{k_{v}} \hat{\mathbf{d}}_{\mathbf{k}} / 2$, where $\hat{\mathbf{d}}_{\mathbf{k}}=\mathbf{d}_{\mathbf{k}} / d_{\mathbf{k}}$ is a unit vector along the SOC field. In comparison, we find $F_{s \mathbf{k}}^{\mu \nu}=s F_{\mathbf{k}}^{\mu \nu}$ with $F_{\mathbf{k}}^{\mu \nu}=\left(\partial_{k_{\mu}} \hat{\mathbf{d}}_{\mathbf{k}} \times \partial_{k_{v}} \hat{\mathbf{d}}_{\mathbf{k}}\right) \cdot \hat{\mathbf{d}}_{\mathbf{k}} / 2$ for the corresponding Berry curvatures, where each one of its components is determined by the quantum metric $\left|F_{\mathbf{k}}^{\mu v}\right|=\left(g_{\mathbf{k}}^{\mu \mu} g_{\mathbf{k}}^{\nu v}-g_{\mathbf{k}}^{\mu v} g_{\mathbf{k}}^{v \mu}\right)^{1 / 2}$ up to a k-dependent sign.

Prior to studying the interplay between the intrahelicity and interhelicity contributions, let us briefly sketch how their tangled sum reproduces the familiar expressions reported in the recent cold-atom literature [28-31]. For this purpose, we first recast the conventional number Eq. (3) via an integration by parts, i.e., $\quad N=-(1 / 2) \sum_{s \mathbf{k}} k_{\nu} \partial_{k_{v}}\left(1-\xi_{s \mathbf{k}} \mathcal{X}_{s \mathbf{k}} / E_{s \mathbf{k}}\right)$, into an equivalent but somewhat unfamiliar form $N=(1 / 2) \sum_{s \mathbf{k}} k_{\nu}\left(\partial \xi_{s \mathbf{k}} / \partial k_{\nu}\right)\left(\Delta^{2} \mathcal{X}_{s \mathbf{k}} / E_{s \mathbf{k}}^{3}+\xi_{s \mathbf{k}}^{2} \mathcal{Y}_{s \mathbf{k}} / 2 T E_{s \mathbf{k}}^{2}\right)$ This alternative expression holds for any $v$ as long as $\Delta \neq 0$, and we attested its accuracy in our numerics as well. Plugging it into Eq. (4) and taking the $\alpha \rightarrow 0$ limit, we find that $\rho_{\mu \nu}^{\text {intra }}=n \delta_{\mu \nu}-\sum_{\mathbf{k}} k_{\mu} k_{\nu} \mathcal{Y}_{\mathbf{k}} / 2 m A T$ reduces to the conventional expression for a continuum Fermi SF [32] and that Eq. (5) vanishes as the helicity bands unite in this limit. This leads to $\rho_{\mu \nu}=n \delta_{\mu \nu}$ at $T=0$, i.e., the entire Fermi gas is a $\mathrm{SF}$ in the ground state as soon as $\Delta \neq 0$. When $\alpha \neq 0$, in order to attain the precise form of the SF density of, e.g., a 2D Fermi gas with Rashba SOC [29], $\rho_{0}=n-(m / A) \sum_{s \mathbf{k}}\left[\alpha\left(\Delta^{2}+\right.\right.$ $\left.\left.\xi_{\mathbf{k}} \xi_{s \mathbf{k}}\right) \mathcal{X}_{s \mathbf{k}} / 4 s k \xi_{\mathbf{k}} E_{s \mathbf{k}}+(k / m+s \alpha)^{2} \mathcal{Y}_{s \mathbf{k}} / 8 T\right]$, where $k=$ $\left(k_{x}^{2}+k_{y}^{2}\right)^{1 / 2}$, we perform yet another integration by parts on the term $\Delta^{2} \sum_{\mathbf{k}}\left(\alpha^{2}+s \alpha k / m\right) \mathcal{X}_{s \mathbf{k}} / E_{s \mathbf{k}}^{3}=s \alpha A k_{c}-$ $s \alpha \sum_{\mathbf{k}} \xi_{s \mathbf{k}} \mathcal{X}_{s \mathbf{k}} / k E_{s \mathbf{k}}-\sum_{\mathbf{k}}\left(\alpha^{2}+s k \alpha / m\right) \xi_{s \mathbf{k}}^{2} \mathcal{Y}_{s \mathbf{k}} / 2 T E_{s \mathbf{k}}^{2} \quad$ in the alternative number equation. Here the ultraviolet $\mathbf{k}$-space cutoff $k_{c}$ cancels out once summed over $s$. Finally, observing that $g_{\mathbf{k}_{\mu \nu}}^{\mu \nu}=\left(k^{2} \delta_{\mu \nu}-k_{\mu} k_{\nu}\right) / 2 k^{4}$ may effectively be replaced with $g_{\mathbf{k}}^{\mu \nu}=\delta_{\mu \nu} / 4 k^{2}$, due to the even sums over $k_{x}$ and $k_{y}$, we eventually arrive at an isotropic tensor $\rho_{\mu \nu}=\rho_{0} \delta_{\mu \nu}$ with the quoted SF density as the prefactor. Similar procedures apply to 3D Fermi SFs with Weyl [30] and Rashba [28] SOCs. In addition, we also verified that all of our numerical results for $\rho_{\mu \nu}$ benchmark perfectly well with the existing literature [28-31].

The significance of the interhelicity contribution (5) to the SF-density tensor seems to have gone entirely unnoticed in 


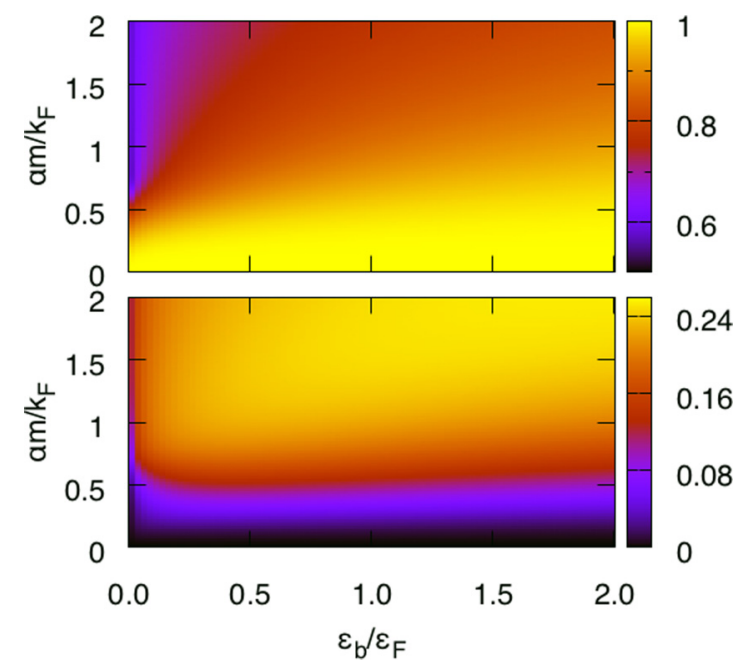

FIG. 1. A 2D Fermi gas with Rashba SOC is mapped at $T=0$ in the plane of two-body binding energy $\epsilon_{b}$ and SOC strength $\alpha$. The total SF fraction $\rho_{0} / n$ is shown on the top along with the overall interhelicity fraction $\rho_{0}^{\text {inter }} / \rho_{0}$ at the bottom.

the physics literature. Having firmly established its geometric origin, ${ }^{1}$ next we explore its relative weight in the whole parameter space starting with a 2D Fermi SF with Rashba SOC.

\section{A. Two-dimensional Fermi gas with Rashba SOC}

In line with the cold-atom literature, here we substitute $U$ with the two-body binding energy $\epsilon_{b} \geqslant 0$ in vacuum via the usual relation $A / U=\sum_{\mathbf{k}} 1 /\left(2 \epsilon_{\mathbf{k}}+\epsilon_{b}\right)$. In addition, we set $n=k_{F}^{2} / 2 \pi$ and define an effective Fermi momentum $k_{F}$ and Fermi energy $\epsilon_{F}=k_{F}^{2} / 2 m$ as the relevant length and energy scales in our numerical calculations. This is in such a way that increasing $U$ from 0 increases $\epsilon_{b}$ continuously from 0 to $\infty$. For instance, a colormap of the ground-state $(T=0) \mathrm{SF}$ density $\rho_{0}=\rho_{0}^{\text {intra }}+\rho_{0}^{\text {inter }}$ is shown in Fig. 1 as functions of $\epsilon_{b}$ and $\alpha$, along with the overall interhelicity fraction $\rho_{0}^{\text {inter }} / \rho_{0}$. See the Appendix for analogous results near the critical BerezinskiiKosterlitz-Thouless (BKT) transition temperature $T=T_{\mathrm{BKT}}$, showing that the thermal effects are quite negligible for most of the parameter regimes of interest.

In the absence of a SOC when $\alpha=0$, Fig. 1 reveals that $\rho_{0}=n$ and $\rho_{0}^{\text {inter }}=0$ for any $\epsilon_{b}>0$, which is a well-known result in the condensed-matter literature [32]. On the other hand, increasing $\alpha$ from 0 gradually depletes the SF fraction down to a saturation value that is eventually determined by the effective mass of the Cooper molecules in the strongcoupling limit, i.e., $\rho_{0} / n \rightarrow 2 m / m_{B}$ for a weakly interacting molecular Bose SF. For instance, it is already known that $m_{B} / m \rightarrow\{2,4\}$ when $m \alpha / k_{F} \rightarrow\{0, \infty\}[29]$ and that the SF fraction exhibits a dip value of $1 / 2$. This is barely seen in

\footnotetext{
${ }^{1}$ Note that the local quantum geometry is non-Abelian as the helicity bands are degenerate for some $\mathbf{k}$ states in the parameter space, e.g., around the origin in our continuum model. However, due to the $\mathbf{k}$ sums, it turns out that the interhelicity contribution to some of the global properties such as the SF density can be expressed in terms of the total quantum metric of the helicity bands as discussed in the text.
}

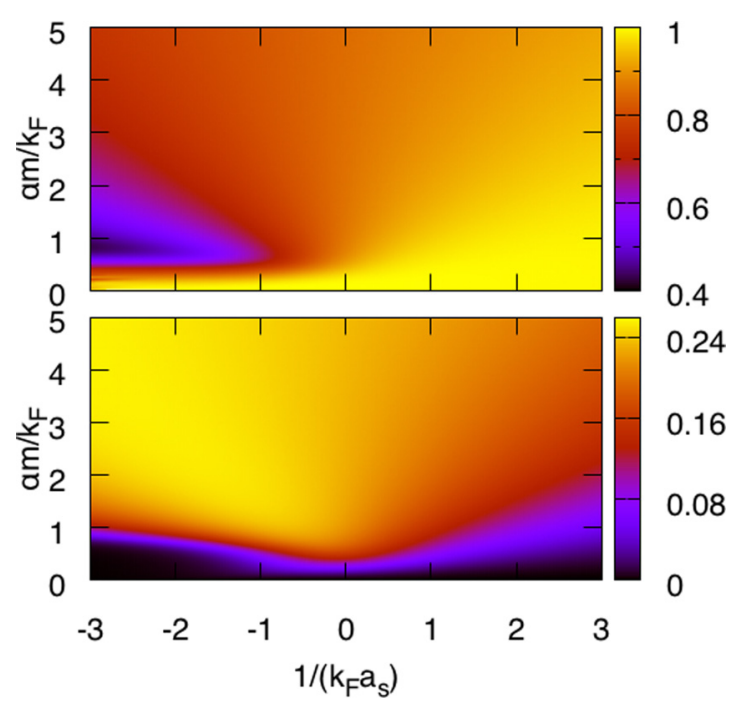

FIG. 2. A 3D Fermi gas with Weyl SOC is mapped at $T=0$ in the plane of two-body scattering length $a_{s}$ and SOC strength $\alpha$. The total SF fraction $\rho_{0} / n$ is shown on the top along with the overall interhelicity fraction $\rho_{0}^{\text {inter }} / \rho_{0}$ on the bottom.

Fig. 1 in a tiny region when $\epsilon_{b} / \epsilon_{F} \rightarrow 0$ for sufficiently large $m \alpha / k_{F}$. More interestingly, increasing $\alpha$ from 0 builds up the overall interhelicity contribution $\rho_{0}^{\text {inter }} / \rho_{0}$, growing slowly to a maximal value of 0.26 for the parameters shown. Furthermore, even though it is not visible here in a $2 \mathrm{D}$ system, as a direct outcome of the competition between the intrahelicity and interhelicity contributions, $\rho_{0}$ evolves nonmonotonically with $\alpha$ especially when $\epsilon_{b} / \epsilon_{F} \ll 1$. Such an interplay is best illustrated in a $3 \mathrm{D}$ Fermi gas as we discuss next.

\section{B. Three-dimensional Fermi gas with Weyl or Rashba SOC}

In line with the cold-atom literature, here we substitute $U$ with the two-body scattering length $a_{s}$ in vacuum via the usual relation $V / U=-m V / 4 \pi a_{s}+\sum_{\mathbf{k}} 1 / 2 \epsilon_{\mathbf{k}}$ and choose $n=k_{F}^{3} / 3 \pi^{2}$ to define the relevant length and energy scales for the numerical calculations. This is in such a way that increasing $U$ from 0 changes the dimensionless parameter $1 / k_{F} a_{s}$ continuously from $-\infty$ to 0 to $+\infty$, for which $\left|a_{s}\right| \rightarrow$ $\infty$ is commonly referred to as the unitarity. For instance, a colormap of the ground-state SF density $\rho_{0}=\rho_{0}^{\text {intra }}+\rho_{0}^{\text {inter }}$ is shown for the Weyl SOC in Fig. 2 as a function of $1 / a_{s}$ and $\alpha$, along with the overall interhelicity fraction $\rho_{0}^{\text {inter }} / \rho_{0}$.

First of all, it is again already known that $m_{B} / m \rightarrow$ $\{2,2.32,6\}$ when $1 / m \alpha a_{s} \rightarrow\{+\infty, 0,-\infty\} \quad[30,37]$ and therefore we expect the depletion of the SF fraction $\rho_{0} / n$ to saturate around 0.34 when $1 / k_{F} a_{s} \ll 0$ and around 0.86 at unitarity for sufficiently large $m \alpha / k_{F}$. Our numerical results shown in Fig. 2 nicely recover these limits. In addition, similar to a 2D Fermi SF, we find that the overall interhelicity contribution $\rho_{0}^{\text {inter }} / \rho_{0}$ builds again up to a maximal value of 0.26 for the parameters shown. Furthermore, the rapid growth of $\rho_{0}^{\text {inter }}$ on the BCS side of the unitarity leads to a nonmonotonic evolution of $\rho_{0}$ with $\alpha$, which is clearly visible in a broad region when $1 / k_{F} a_{s} \lesssim 0$.

For completeness, we also present a colormap of the in-plane ground-state SF density $\rho_{\perp}=\rho_{\perp}^{\text {intra }}+\rho_{\perp}^{\text {inter }}$ for the 


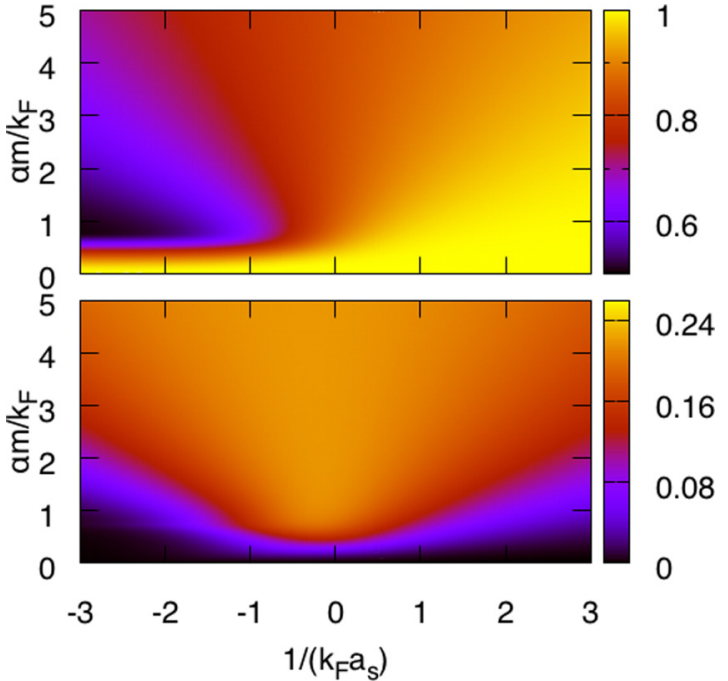

FIG. 3. A 3D Fermi gas with Rashba SOC is mapped at $T=0$ in the plane of two-body scattering length $a_{s}$ and SOC strength $\alpha$. The total SF fraction $\rho_{\perp} / n$ is shown on the top along with the overall interhelicity fraction $\rho_{\perp}^{\text {inter }} / \rho_{\perp}$ on the bottom.

Rashba SOC in Fig. 3, along with the overall interhelicity fraction $\rho_{\perp}^{\text {inter }} / \rho_{\perp}$. Note that $\rho_{z z}=n$ and $\rho_{z z}^{\text {inter }}=0$ for the entire parameter space at $T=0$ and are not shown. Our numerical results are again in perfect agreement with the expected results, for which we already know that $m_{B} / m \rightarrow$ $\{2,2.40,4\}$ when $1 / m \alpha a_{s} \rightarrow\{+\infty, 0,-\infty\}$ [28,37], leading $\rho_{0} / n$ to saturate around 0.5 when $1 / k_{F} a_{s} \ll 1$ and around 0.84 at unitarity for sufficiently large $m \alpha / k_{F}$. In addition, the overall interhelicity contribution $\rho_{0}^{\text {inter }} / \rho_{0}$ builds up to a maximal value of 0.22 for the parameters shown. Thus, in comparison to the Weyl SOC shown in Fig. 2, $\rho_{0}^{\text {inter }}$ is slightly weaker here on the BCS side of the unitarity, even though it is rather comparable on the BEC side. As the 2D SOCs have recently been created with atomic Bose and Fermi gases [33-36], our predictions in this paper may already be verified in similar setups. Noting that the quantum metric effects have so far proved to be quite rare and elusive in condensed-matter physics [12-25], in contrast to the Berry curvature ones that are ubiquitously found in nature [2-9], there is no doubt that its cold-atom realization will be one of the landmark breakthroughs in modern quantum physics.

\section{CONCLUSION}

In summary, while having primarily focused on the spinorbit-coupled atomic Fermi SF but not necessarily limited to it, i.e., in the much broader context of superfluidity and superconductivity, here we examined the prospects for revealing the quantum geometry of the noninteracting helicity bands through measuring the SF density of the system. For this purpose, we first divided the SF-density tensor into two contributions based on their physical origin, i.e., while the intrahelicity contribution has the conventional form determined by the helicity spectrum, the interhelicity one has a geometric origin related to the total quantum metric of the helicity bands. We then considered both Rashba and Weyl SOCs across the BCS-BEC crossover and showed that the geometrical contribution accounts for up to a quarter of the total SF density.

Thus, by studying the competition between the intraband and interband contributions to the SF density, as well as the hidden role played by the quantum metric, our extensive numerical calculations on spin-orbit-coupled Fermi gases exposed the missing link between the nonmonotonic evolution of the SF density and the quantum geometry of the helicity bands. This is our main finding in this paper. In addition, our work also shed light on the underlying physical mechanism behind other nonmonotonic effects as the SF density is directly related to the mass of the SF carriers. For instance, in the follow-up studies [38-40], we have recently showed that the quantum metric governs not only the SF density but also many other observables, including the sound velocity and spin susceptibility, through renormalizing the effective mass of the two-body bound states and Cooper pairs in general.

Incentivized by the recent creations of 2D SOCs along with the ongoing push toward simulating diverse aspects of spin-orbit physics in the cold-atom community [33-36], we believe realization of such a geometric effect will be one of the long-standing milestones in modern quantum many-body physics, where not only the topology but also the geometry of the underlying band structure plays an ever-increasing role. As a possible probe, we expect nonmonotonic evolutions for those SF (normal-state) properties that are inversely proportional to the effective mass of the SF carriers (preformed pairs).

\section{ACKNOWLEDGMENTS}

The author acknowledges support from TÜBİTAK and the BAGEP award of the Turkish Science Academy.

\section{APPENDIX: THE BKT TRANSITION TEMPERATURE}

The BKT transition temperature is determined by the universal BKT relation $T_{\mathrm{BKT}}=\frac{\pi}{8 m} \sqrt{\rho_{x x} \rho_{y y}-\rho_{x y} \rho_{y x}}=$ $\pi \rho_{0} / 8 m$, self-consistently with the mean-field order parameter

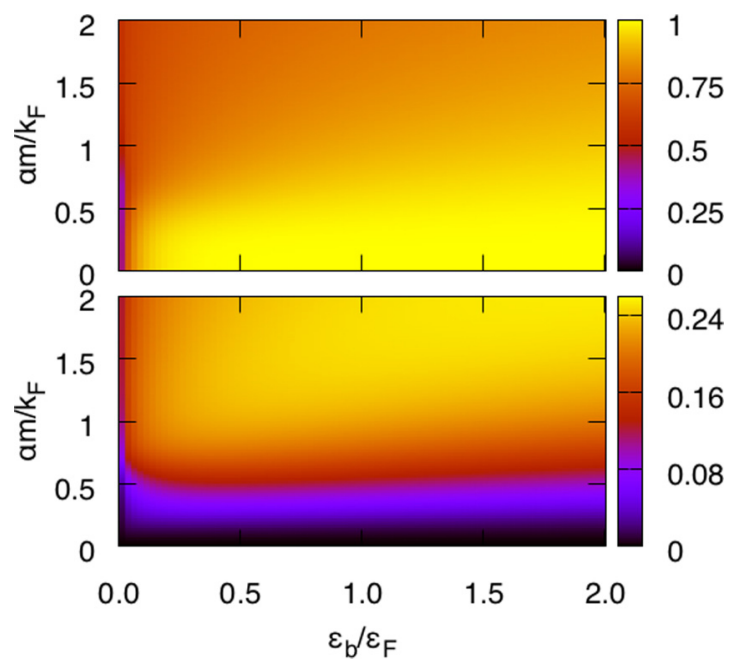

FIG. 4. A 2D Fermi gas with Rashba SOC is mapped at $T=T_{\mathrm{BKT}}$ in the plane of two-body binding energy $\epsilon_{b}$ and SOC strength $\alpha$. The total SF fraction $\rho_{0} / n$ is shown at the top along with the overall interhelicity fraction $\rho_{0}^{\text {inter }} / \rho_{0}$ at the bottom. 
$\Delta$ and the corresponding chemical potential $\mu$. As shown in Fig. 4, the geometric effect remains intact even at $T=T_{\mathrm{BKT}}$ and it is very much similar to that of the ground state that is shown in Fig. 1.
[1] M. V. Berry, in The Quantum Phase, Five Years after in Geometric Phases in Physics, edited by A. Shapere and F. Wilczek (World Scientific, Singapore, 1989).

[2] D. J. Thouless, Topological Quantum Numbers in Nonrelativistic Physics (World Scientific, Singapore, 1998).

[3] D. Xiao, M.-C. Chang, and Q. Niu, Berry phase effects on electronic properties, Rev. Mod. Phys. 82, 1959 (2010).

[4] M. Z. Hasan and C. L. Kane, Colloquium: Topological insulators, Rev. Mod. Phys. 82, 3045 (2010).

[5] X.-L. Qi and S.-C. Zhang, Topological insulators and superconductors, Rev. Mod. Phys. 83, 1057 (2011).

[6] J. Sinova, S. O. Valenzuela, J. Wunderlich, C. H. Back, and T. Jungwirth, Spin Hall effects, Rev. Mod. Phys. 87, 1213 (2015).

[7] C.-K. Chiu, J. C. Y. Teo, A. P. Schnyder, and S. Ryu, Classification of topological quantum matter with symmetries, Rev. Mod. Phys. 88, 035005 (2016).

[8] A. Bansil, H. Lin, and T. Das, Colloquium: Topological band theory, Rev. Mod. Phys. 88, 021004 (2016).

[9] F. D. M. Haldane, Nobel lecture: Topological quantum matter, Rev. Mod. Phys. 89, 040502 (2017).

[10] See, for instance, D. C. Brody and L. P. Hughston, Geometric quantum mechanics, J. Geom. Phys. 38, 19 (2001).

[11] J. P. Provost and G. Vallee, Riemannian structure on manifolds of quantum states, Commun. Math. Phys. 76, 289 (1980).

[12] F. D. M. Haldane, Geometrical Description of the Fractional Quantum Hall Effect, Phys. Rev. Lett. 107, 116801 (2011).

[13] T. Neupert, C. Chamon, and C. Mudry, Measuring the quantum geometry of Bloch bands with current noise, Phys. Rev. B 87, 245103 (2013).

[14] R. Roy, Band geometry of fractional topological insulators, Phys. Rev. B 90, 165139 (2014).

[15] T. S. Jackson, G. Moller, and R. Roy, Geometric stability of topological lattice phases, Nat. Commun. 6, 8629 (2015).

[16] Y. Gao, S. A. Yang, and Q. Niu, Field Induced Positional Shift of Bloch Electrons and its Dynamical Implications, Phys. Rev. Lett. 112, 166601 (2014).

[17] Y. Gao, S. A. Yang, and Q. Niu, Geometrical effects in orbital magnetic susceptibility, Phys. Rev. B 91, 214405 (2015).

[18] A. Srivastava and A. Imamoglu, Signatures of BlochBand Geometry on Excitons: Nonhydrogenic Spectra in Transition-Metal Dichalcogenides, Phys. Rev. Lett. 115, 166802 (2015).

[19] L.-K. Lim, J.-N. Fuchs, and G. Montambaux, Geometry of Bloch states probed by Stückelberg interferometry, Phys. Rev. A 92, 063627 (2015).

[20] F. Piéchon, A. Raoux, J.-N. Fuchs, and G. Montambaux, Geometric orbital susceptibility: Quantum metric without Berry curvature, Phys. Rev. B 94, 134423 (2016).

[21] S. Peotta and P. Törmä, Superfluidity in topologically nontrivial flat bands, Nat. Commun. 6, 8944 (2015).

[22] A. Julku, S. Peotta, T. I. Vanhala, D.-H. Kim, and P. Törmä, Geometric Origin of Superfluidity in the Lieb-Lattice Flat Band, Phys. Rev. Lett. 117, 045303 (2016).
[23] L. Liang, T. I. Vanhala, S. Peotta, T. Siro, A. Harju, and P. Törmä, Band geometry, Berry curvature, and superfluid weight, Phys. Rev. B 95, 024515 (2017).

[24] L. Liang, S. Peotta, A. Harju, and P. Törmä, Wave-packet dynamics of Bogoliubov quasiparticles: Quantum metric effects, Phys. Rev. B 96, 064511 (2017).

[25] M. Iskin, Berezinskii-Kosterlitz-Thouless transition in the timereversal-symmetric Hofstadter-Hubbard model, Phys. Rev. A 97, 013618 (2018).

[26] N. B. Kopnin and E. B. Sonin, BCS Superconductivity of Dirac Electrons in Graphene Layers, Phys. Rev. Lett. 100, 246808 (2008).

[27] N. B. Kopnin and E. B. Sonin, Supercurrent in superconducting graphene, Phys. Rev. B 82, 014516 (2010).

[28] K. Zhou and Z. Zhang, Opposite Effect of Spin-Orbit Coupling on Condensation and Superfluidity, Phys. Rev. Lett. 108, 025301 (2012).

[29] L. He and X.-G. Huang, BCS-BEC Crossover in 2D Fermi Gases with Rashba Spin-Orbit Coupling, Phys. Rev. Lett. 108, 145302 (2012).

[30] L. He and X.-G. Huang, BCS-BEC crossover in threedimensional Fermi gases with spherical spin-orbit coupling, Phys. Rev. B 86, 014511 (2012).

[31] M. Gong, G. Chen, S. Jia, and C. Zhang, Searching for Majorana Fermions in 2D Spin-Orbit Coupled Fermi Superfluids at Finite Temperature, Phys. Rev. Lett. 109, 105302 (2012).

[32] P. J. H. Denteneer, G. An, and J. M. J. van Leeuwen, Helicity modulus in the two-dimensional Hubbard model, Phys. Rev. B 47, 6256 (1993).

[33] Z. Wu, L. Zhang, W. Sun, X.-T. Xu, B.-Z. Wang, S.-C. Ji, Y. Deng, S. Chen, X.-J. Liu, and J.-W. Pan, Realization of twodimensional spin-orbit coupling for Bose-Einstein condensates, Science 354, 83 (2016).

[34] W. Sun, B.-Z. Wang, X.-T. Xu, C.-R. Yi, L. Zhang, Z. Wu, Y. Deng, X.-J. Liu, S. Chen, and J.-W. Pan, Long-lived 2D spinorbit coupled topological Bose gas, arXiv:1710.00717.

[35] L. Huang, Z. Meng, P. Wang, P. Peng, S.-L. Zhang, L. Chen, D. Li, Q. Zhou, and J. Zhang, Experimental realization of a two-dimensional synthetic spin-orbit coupling in ultracold Fermi gases, Nat. Phys. 12, 540 (2016).

[36] Z. Meng, L. Huang, P. Peng, D. Li, L. Chen, Y. Xu, C. Zhang, P. Wang, and J. Zhang, Experimental Observation of a Topological Band Gap Opening in Ultracold Fermi Gases with Two-Dimensional Spin-Orbit Coupling, Phys. Rev. Lett. 117, 235304 (2016).

[37] M. Iskin and A. L. Subaşı, Quantum phases of atomic Fermi gases with anisotropic spin-orbit coupling, Phys. Rev. A 84, 043621 (2011).

[38] M. Iskin, Quantum metric contribution to the pair mass in spinorbit coupled Fermi superfluids, Phys. Rev. A 97, 033625 (2018).

[39] M. Iskin, Spin susceptibility of spin-orbit coupled Fermi superfluids, Phys. Rev. A 97, 053613 (2018).

[40] M. Iskin, Geometric mass acquisition via quantum metric: An effective band mass theorem for the helicity bands, arXiv:1803.04176. 